\title{
Comparative Efficacy of Fentanyl and Morphine in Patients with or At Risk for Acute Respiratory Distress Syndrome: A Propensity Score-Matched Cohort Study
}

\author{
An-Min Hu${ }^{1}$ [D $\cdot$ Zhi-Ming Shan ${ }^{1} \cdot$ Zhong-Jun Zhang $^{1} \cdot$ Hui-Ping Li ${ }^{2}$
}

Accepted: 28 January 2021 / Published online: 19 April 2021

(c) The Author(s) 2021

\begin{abstract}
Introduction Opioids are potent painkillers but can have severe adverse effects in the intensive care unit (ICU). The aim of this study was to compare the outcomes of fentanyl and morphine use among patients at risk for and with acute respiratory distress syndrome (ARDS).

Methods We developed a dataset of real-world data to enable the comparison of the effectiveness and safety of opioids and the associated outcomes from the Multiparameter Intelligent Monitoring in Intensive Care (MIMIC)-III database and the eICU Collaborative Research Database. Patients who were admitted to the ICU with a diagnosis of or at risk for ARDS and received mechanical ventilation for at least $12 \mathrm{~h}$ were included. Patients were enrolled sequentially into one of six groups in three cohorts: treated with fentanyl or not; treated with morphine or not; and treated with fentanyl or morphine. Propensity score matching and multivariable analyses were performed.

Results Fentanyl was associated with higher in-hospital mortality in the propensity score-matched model but not in the linear regression model. The use of morphine was associated with a higher in-hospital mortality in both models. Both fentanyl and morphine were associated with longer duration of mechanical ventilation, ICU stay, and hospitalization and a decreased likelihood of being discharged home in both models. Notably, compared with morphine, fentanyl was associated with a lower mortality and an increased likelihood of being discharged home.

Conclusions Both fentanyl and morphine were independent risk factors for worse outcomes in patients with or at risk for ARDS. Compared with morphine, fentanyl may be preferred in these patients.
\end{abstract}

\section{Introduction}

Pain is common among patients admitted to intensive care units (ICUs), with a prevalence of $40-77 \%$ [1]. Severe pain negatively affects the status of critically ill adults, resulting in prolonged mechanical ventilation, respiratory injury, and immunosuppression [2, 3]. In addition, the use of opioids is associated with a significant risk of mortality in a dosedependent manner [4-8].

Hui-Ping Li

Li.huiping@szhospital.com

Department of Anesthesiology, Shenzhen People's Hospital, Shenzhen, China

2 Department of Respiratory and Critical Care Medicine, Shenzhen People's Hospital, No. 1017 Dongmen North Road, Shenzhen 518020, Guangdong, China
Acute respiratory distress syndrome (ARDS) is the cause of respiratory failure in $10.4 \%$ of critically ill patients and has a mortality rate of approximately $40 \%$ [9-11]. Clinical guidelines suggest that intravenous opioids be considered as the first-line drug to manage pain in critically ill patients [12, 13]. It is unclear whether patients treated with fentanyl and morphine have similar outcomes. To address this gap, we systematically examined the association between outcomes and the use of opioids (fentanyl and morphine) in patients with or at risk for ARDS. Our study provided a comprehensive analysis across populations, hospital characteristics, and outcomes. 


\section{Key Points}

Both fentanyl and morphine were independently risk factors in patients with or at risk for ARDS.

Opioids were increased in the duration of mechanical ventilation, ICU stay and hospital stay.

Fentanyl was associated with lower mortality rate and higher rate of discharge home than morphine.

Fentanyl may be superior to morphine for a sedative in patients with or at risk for ARDS.

\section{Methods}

\subsection{Data Source}

The Multiparameter Intelligent Monitoring in Intensive Care (MIMIC) III (version 1.4) database and eICU Collaborative Research Database are maintained by the Laboratory for Computational Physiology at the Massachusetts Institute of Technology in the US $[14,15]$. The database is accessible to researchers who have passed a training course on protecting human subjects. The data were extracted by AMH (certification number: 26450451).

\subsection{Study Population and Stratification}

Mechanically ventilated ICU patients with a diagnosis of ARDS or known ARDS risk factors were included. ARDS risk factors included acute hypoxemic respiratory failure, pneumonia, sepsis, trauma, burns, and other diagnoses or treatments (i.e., multiple transfusions) [9, 10, 16, 17]. Patient diagnoses were determined on the basis of the International Classification of Disease Codes, Ninth Revision, Clinical Modification (ICD-9-CM) (supplement file 1, see electronic supplementary material [ESM]) [18]. Comorbidities were extracted according to the Elixhauser Comorbidity Index based on the diagnoses recorded during hospitalization [19].

The inclusion criteria in this study were as follows: (1) for patients with multiple ICU stays, only the first ICU stay was eligible; (2) adults ( $\geq 18$ years of age) on ICU admission; (3) ICU stay $\geq 24 \mathrm{~h}$; and (4) the use of invasive mechanical ventilation for at least $12 \mathrm{~h}$.

\subsection{Outcomes}

The following outcome measures were assessed: hospital mortality, ventilation duration, ICU length of stay, hospitalization duration, and discharge destination (home versus elsewhere).

\subsection{Data Analysis}

The considered factors were age; sex; ethnicity; weight; height; acute physiology and chronic health evaluation (APACHE)-III score; oxygenation index; alveolar-arterial oxygen difference (AaDo2); diagnoses at discharge; hospital characteristics; the use of sedatives and opioids. The APACHE-III scoring system is designed to prospectively predict mortality in individual ICU patients. Each patient had a diagnosis of ARDS or a known ARDS risk factor at the time of admission [20]. Patient diagnoses at discharge included the following: ARDS, pneumonia, sepsis, aspiration, heart failure, chronic obstructive pulmonary disease (COPD), disseminated intravascular coagulation (DIC), liver disease, renal failure, hypertension, and diabetes mellitus. Hospital characteristics were defined in the database, including ICU type, number of ICU beds, teaching status, and provider region. Sedatives and opioids included the following: midazolam, propofol, dexmedetomidine, and fentanyl.

Patients were enrolled sequentially into three cohorts: did or did not use morphine; did or did not use fentanyl; and used morphine or fentanyl. Descriptive data are presented as the median (25th to 75th percentile) for continuous variables and frequency (\%) for categorical variables. Categorical variables were compared between groups using the chi-square test. An unpaired $t$ test or Kruskal-Wallis test was used for continuous variables.

Propensity score generation, stratification by deciles, and 1:1 matching between groups were performed using the $\mathrm{R}$ package MatchIt [21]. A non-parsimonious regression model was used to produce propensity scores for the group with fewer patients using the patient characteristics described above. For the propensity score-matched analysis (primary analysis), each patient in the group with fewer patients was matched to the third decimal point using the nearest neighbor algorithm. A caliper setting of 0.05 was utilized. Standardized differences (SDs) were used to confirm a balanced matching result. The matching result was considered balanced when the SD was $<0.1$. The final models included each hospital as a random effect and all patient characteristics used to calculate the propensity score. Additionally, multivariable regression, including all the patient characteristics used to calculate the propensity score, was used to confirm the findings (secondary analysis).

The following pre-specified subgroups and interactions were assessed: age (within 18-65 years, and 65 years or older), duration of mechanical ventilation (12-24 h, 24-48 h, and $48 \mathrm{~h}$ or longer), and using first-line sedation (dexmedetomidine, midazolam, propofol). 
Missing data were imputed with the multivariate imputation by chained equations (MICE) method [22]. The amount of missing data was low, and the missing data were detailed in supplemental file 2 (see ESM). Supplemental file 3 showed the frequency of missing data elements and the distribution of each parameter before and after imputation (see ESM). All analyses were performed using R version 3.62.

\section{Results}

This study was conducted and reported in accordance with Strengthening the Reporting of Observational Studies in Epidemiology (STROBE) guidelines (supplemental file 4, see ESM) [23]. In total, 46,428 ICU patients and 61,051 ICU admissions were available in the MIMIC III database v1.4, and 177,863 ICU patients and 626,858 ICU admissions were available in the eICU Collaborative Research Database. Sequentially, we excluded 8433 patients whose age at admission was younger than 18 years, 116,599 patients who stayed in the ICU for $<24 \mathrm{~h}$, and 81,849 patients who received mechanical ventilation for $<12 \mathrm{~h}$, as shown in supplemental file 5 (see ESM). The included 17,410 patients had at least one ARDS risk factor: 12,567 patients were diagnosed with acute hypoxemic respiratory failure, 4517 patients were diagnosed with pneumonia, 4757 patients were diagnosed with sepsis, and 1603 patients were diagnosed with aspiration.

A total of 5515 patients (31.7\%) received fentanyl, and $601(3.5 \%)$ received morphine. Before propensity score matching, there were statistically significant differences in admission type in the analysis of patients who did and did not receive fentanyl, those who did and did not receive morphine, and those who received fentanyl or morphine. Overall, patients who received fentanyl or morphine had a lower oxygenation index, had a higher AaDo2 and were more likely to be female and to be diagnosed with ARDS, pneumonia, sepsis, aspiration, heart failure, hypertension, and diabetes than their counterparts (supplemental files 6-7, see
ESM). New users of fentanyl had a lower oxygenation index, higher APACHE III score, and higher AaDo2 and were more likely to be diagnosed with ARDS, aspiration, and hypertension than patients who received morphine (supplemental file 8, see ESM). However, propensity score matching yielded an adequate covariate balance, reducing concerns that the measured effects were affected by baseline confounders.

\subsection{Propensity-Matched Analysis}

The fully adjusted, propensity score-matched analysis of the outcomes was shown in Table 1. Patients who were treated with fentanyl had a higher mortality rate than patients not treated with fentanyl (OR 1.30, 95\% CI 1.13-1.49; $p<$ 0.001); likewise, patients treated with morphine had a higher mortality rate than patients not treated with morphine (OR 6.70, 95\% CI 4.95-9.13; $p<0.001)$. Unexpectedly, patients who were treated with fentanyl had a lower mortality rate than patients treated with morphine (OR $0.16,95 \%$ CI $0.10-0.25 ; p<0.001)$.

Patients who received fentanyl had a longer ventilation duration, ICU stay, and hospital stay than those who did not receive fentanyl $(p<0.001)$. Similarly, patients who used morphine had a longer ventilation duration $(p<0.01)$, ICU stay $(p<0.001)$, and hospital stay $(p<0.001)$ than those who did not use morphine. The comparison of patients who used fentanyl and morphine showed that there were no differences in ventilation duration $(p=0.44)$, ICU stay $(p=0.14)$, or hospital stay $(p=0.03)$.

Patients treated with fentanyl had a lower rate of discharge home than those who did not receive fentanyl (OR $0.73,95 \%$ CI $0.65-0.82 ; p<0.001)$. The use of morphine also resulted in a lower rate of discharge home (OR 0.17, 95\% CI 0.11-0.25; $p<0.001)$. Furthermore, patients who were treated with fentanyl had a higher rate of discharge home than patients who were treated with morphine.

Table 1 Results of propensity-matched analysis in patients with or at risk for acute respiratory distress syndrome

\begin{tabular}{lllcccc}
\hline & Comparator & Hospital mortality* & Ventilator days ${ }^{\dagger}$ & ICU days $^{\dagger}$ & Hospital days $^{\dagger}$ & Discharge to home* $^{*}$ \\
\hline Fentanyl & No fentanyl & $1.30(1.13-1.49),<$ & $0.35(0.22-0.48),<$ & $1.86(1.50$ to 2.24$),<2.21(1.33-3.09),<$ & $0.73(0.65-0.82),<$ \\
& & 0.001 & 0.001 & 0.001 & 0.001 & 0.001 \\
Morphine & No morphine & $6.70(4.95-9.13),<$ & $-0.55(-0.90$ to & $10.85(8.80$ to 12.42$), 8.24(5.85-10.64),<$ & $0.17(0.11-0.25),<$ \\
& & 0.001 & $-0.20),<0.01$ & $<0.001$ & 0.001 & 0.001 \\
Fentanyl & Morphine & $0.16(0.10-0.25),<$ & $0.16(-0.25$ to 0.57$),-1.21(-2.83$ to & $2.56(0.20-4.92), 0.03$ & $3.53(2.09-6.14),<$ \\
& & 0.001 & 0.44 & $0.40), 0.14$ & 0.001 & \\
\hline
\end{tabular}

Dex dexmedetomidine

*Data are presented as odds ratio (95\% confidence interval), $p$ value

${ }^{\dagger}$ Data are presented as difference of variable value (95\% confidence interval), $p$ value 
Table 2 Results of multivariate analysis in patients with or at risk for acute respiratory distress syndrome

\begin{tabular}{lllllll}
\hline & Comparator & Hospital mortality* & Ventilator days ${ }^{\dagger}$ & ICU days $^{\dagger}$ & Hospital days $^{\dagger}$ & Discharge to home* $^{*}$ \\
\hline Fentanyl & No Fentanyl & $1.02(0.94-1.11), 0.60$ & $0.15(0.06$ to 0.23$),<$ & $2.34(2.05$ to 2.64$),<2.34(1.78-2.90),<$ & $0.81(0.75-0.87),<$ \\
& & & 0.001 & 0.001 & 0.001 & 0.001 \\
Morphine & No Morphine & $5.69(4.73-6.87),<$ & $-0.58(-0.79$ to & $11.5(10.7$ to 12.2$),<9.15(7.77-10.5),<$ & $0.19(0.14-0.26),<$ \\
& & 0.001 & $-0.37),<0.001$ & 0.001 & 0.001 & 0.001 \\
Fentanyl & Morphine & $0.11(0.08-0.15),<$ & $0.39(0.02$ to 0.76$)$, & $-0.70(-1.79$ to & $2.72(0.64-4.80), 0.013 .43(2.31-5.27),<$ \\
& & 0.001 & 0.04 & $0.38), 0.20$ & 0.001 & 0 \\
\hline
\end{tabular}

Dex dexmedetomidine

*Data are presented as odds ratio (95\% confidence interval), $p$ value

${ }^{\dagger}$ Data are presented as difference of variable value (95\% confidence interval), $p$ value

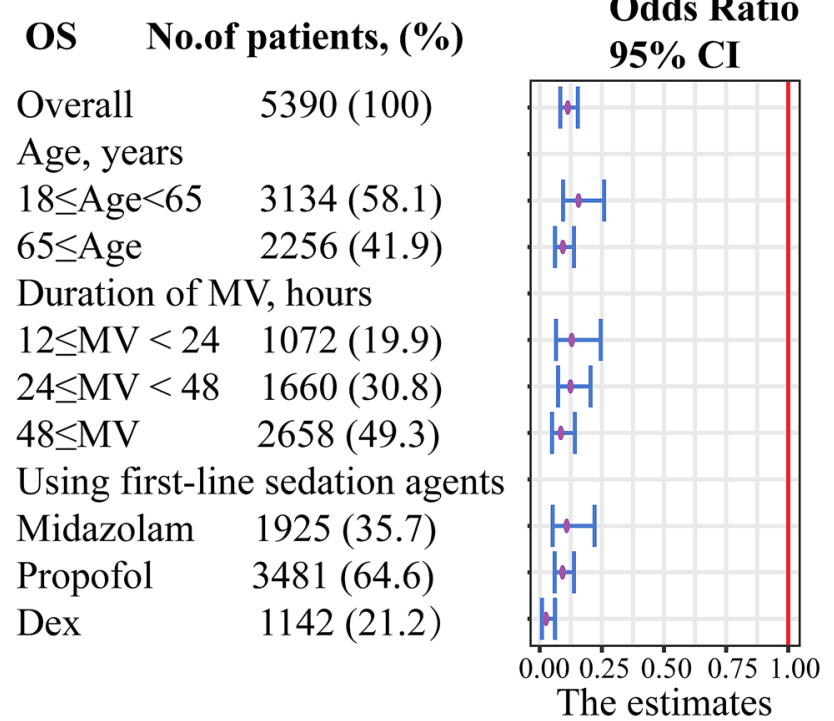

Fig. 1 Forest plot evaluating the impact of hospital mortality in fentanyl and morphine ( $M V$ mechanical ventilation)

\subsection{Multivariable Analysis}

This multivariable analysis of the five outcomes in patients treated with fentanyl and morphine was shown in Table 2. There was no difference in the mortality rate between patients who did and did not receive fentanyl (OR 1.02, 95\% CI $0.94-1.11 ; p=0.60)$, but patients treated with fentanyl had a lower mortality than those treated with morphine (OR $0.11,95 \%$ CI $0.08-0.15 ; p<0.001)$. Furthermore, fentanyl maintained decreased hospital mortality with respect to age, duration of mechanical ventilation, and sedative agent factor compared with morphine (Fig. 1). Patients treated with morphine also had a higher mortality rate than patients who did not receive morphine (OR 5.69, 95\% CI 4.73-6.87; $p<$ 0.001) (Table 2).

When compared with patients who were not treated with morphine or fentanyl, patients treated with fentanyl or morphine had a longer ventilation duration, ICU stay, and hospitalization duration $(p<0.001)$. Compared with morphine, fentanyl treatment was associated with a significantly longer ventilation duration $(p=0.04)$ and hospital stay $(p=0.01)$ but not ICU stay $(p=0.20)$.

Patients treated with fentanyl had a lower likelihood of being discharged home than patients who were not treated with fentanyl (OR $0.81,95 \%$ CI $0.75-0.87 ; p<0.001$ ) but a higher rate of discharge home than patients treated with morphine (OR 3.43, 95\% CI 2.31-5.27; $p<0.001$ ).

\section{Discussion}

We performed a multicenter, observational cohort study to assess outcomes in patients at risk for or with ARDS who were treated with fentanyl or morphine. We showed that patients treated with fentanyl did not have a significantly elevated mortality rate in the linear regression model but had a significantly elevated in-hospital mortality rate in the propensity score-matched model. The use of morphine was associated with an elevated in-hospital mortality rate. Interestingly, patients treated with fentanyl had a significantly lower mortality rate and were more likely to be discharged home than patients treated with morphine. Additionally, when compared with patients who were not treated with either agent, the use of fentanyl or morphine was associated with a longer ventilator duration, ICU stay, and hospital stay and a reduced likelihood of being discharged home.

A prior case-cohort study showed that the risk of drugrelated adverse events among individuals treated for chronic non-cancer pain with opioids was elevated at opioid dosages equivalent to $\geq 50 \mathrm{mg}$ /day morphine [4,5]. Similarly, patients prescribed a long-acting opioid for chronic noncancer pain had a risk of all-cause mortality that was 1.64 times greater than that of matched patients treated with an analgesic anticonvulsant or a low-dose cyclic antidepressant [6]. Moreover, the administration of morphine early after 
admission was associated with elevated risks of in-hospital mortality and the need for mechanical ventilation in 13,788 patients with acute heart failure in a separate study [7]. A recent cohort study showed that chronic opioid use was associated with an excess risk of mortality in the 6-18 months after traumatic injury [8].

There are also multiple mechanisms by which opioids could increase the risk of all-cause mortality. Opioids can depress the respiratory drive and exacerbate sleep-disordered breathing [24-26]. Additionally, long-term use is also associated with increased myocardial infarction and immunosuppression $[27,28]$. The results of the present study show that the use of fentanyl or morphine in patients with or at risk for ARDS has adverse effects on the prognosis of the patients. In addition, we observed remarkable increases in the duration of mechanical ventilation, ICU stay and hospital stay after opioid administration.

The effects of fentanyl and morphine on the incidence of pain assessment did not differ in patients who were treated for suspected ischemic chest pain in the pre-hospital setting [29], or in patients who were undergoing off-pump coronary artery bypass surgery [30]. However, the administration of morphine was associated with a significantly reduced release of inflammatory cytokines, a greater inhibition of adhesion molecule expression, and a lower incidence of postoperative hyperthermia compared with the administration of fentanyl [31]. Furthermore, fentanyl and morphine differ in terms of the signal transduction mechanism underlying the antinociceptive effects [32,33] and the induction of immunosuppression in animal models [34]. Unfortunately, systematic comparisons of the efficacy and safety of fentanyl and morphine are still lacking. Our findings suggest that fentanyl may be superior to morphine in patients at risk for and with ARDS.

This study took advantage of disparate ICU databases across a range of hospital and ICU settings. These largescale and unfiltered populations represent real-world practice better than the restricted study populations in prescribed treatment and follow-up settings in randomized controlled trials (RCTs). Meanwhile, this study systematically evaluated several key variables in critically ill patients. These factors have not generally been assessed together in other opioid studies. Although fentanyl was the drug of choice for patients with relatively more severe disease (supplement files 7-8, see ESM), and patients treated with fentanyl had a lower oxygenation index, higher $\mathrm{AaDo} 2$, and higher rate of ARDS than patients treated with morphine, our study has observed a lower mortality rate in patients treated with fentanyl than in those treated with morphine.

However, our study had some limitations. First, we only evaluated the outcomes in patients treated with fentanyl and morphine. There were also some missing values for multiple confounding variables that could not be effectively merged or compared, such as analgesic dosage, treatment duration, and daily pain assessment data. Bias may have remained despite the use of propensity score matching and regression to control for a variety of patient and hospital confounders. Second, the proportion of patients treated with morphine was low, and propensity score matching and linear regression analyses included only a subset of the patients in the databases from the United States. Furthermore, we cannot exclude the possibility of subpopulations not sufficiently captured in our different cohorts that could have had considerably different effectiveness profiles. Thus, our results should be cautiously applied to all patients at risk for or with ARDS. Third, our findings potentially lead to both a practical and ethical dilemma. However, patients' comfort remains an important treatment goal. It would be reasonable to treat pain as well as to consider the potential adverse effects of opioid therapy on ARDS outcomes and overall survival. A delicate balance needs to be achieved and maintained between pain treatment and avoiding the negative effects of opioids on the outcomes of patients with ARDS. The use of non-opioid analgesics could decrease the amount of opioids administered and decrease opioid-related side effects in critically ill adults $[12,13]$. Fourth, patients who were included as eligible participants with $\geq 24 \mathrm{~h} \mathrm{ICU}$ stay and who received at least $12 \mathrm{~h}$ ' mechanical ventilation were considered a cohort with more severe illness than the general population. It is possible that part of the reduction in ICU stay or ventilator time is associated with non-statistically significant differences in mortality between groups [35-37]. Fifth, propensity matching results in studying only a subset of the population, which may limit generalizability. Finally, this is an observational study and thus causal associations cannot be determined.

\section{Conclusion}

The use of fentanyl or morphine was independently associated with negative outcomes in patients at risk for and with ARDS. Interestingly, the use of fentanyl was associated with a lower mortality rate and a higher rate of discharge home than the use of morphine. Therefore, fentanyl may be the opioid analgesic of choice for these patients. Further trials are needed to evaluate the mechanism underlying these differences and to validate these findings in other cohorts of patients.

Supplementary Information The online version contains supplementary material available at https://doi.org/10.1007/s40268-021-00338-3.

Acknowledgements The authors thank the patients for their participation. 


\section{Declarations}

Funding Dr. Hu received funding from the National Science Foundation for Young Scientists of China (81801947). Dr. Shan received funding from Shenzhen Natural Science Foundation (JCYJ20190807154401665). Dr. Li received support for article research from Shenzhen Key Medical Discipline Construction Fund.

Conflict of Interest The authors declare that they have no competing interests.

Ethics Approval Consent was obtained for the original data collection, and the institutional review boards of the Massachusetts Institute of Technology (Cambridge, MA, USA) approved the establishment of the database. Therefore, the ethical approval statement and informed consent were waived for this manuscript.

Consent to Participate Not applicable.

Consent for Publication Not applicable.

Availability of Dta and Material The two databases used in this research, MIMIC-III and eICU, are available for access, in part or in total, by relevant parties subject to abiding by their usage policies. To facilitate the reproduction of our results, we shall make fully anonymized data available on figshare (https://figshare.com/s/f94a6 $79 \mathrm{cc} 5 \mathrm{a} 69 \mathrm{dfcd} 3 \mathrm{bc}$ ) on publication of this manuscript.

Cosadde Availability The databases were queried using Navicat Premium 15.0.21, and computations were implemented in $\mathrm{R}$ version 3.62. Additionally, interested researchers can contact $\mathrm{Mr} \mathrm{Hu}$ via email (anmin.edu@gmail.com) for more detailed information.

Author Contributions According to the guidelines of the International Committee of Medical Journal Editors (ICMJE), all authors contributed to the four criteria. AMH and HPL conceived and designed the study. AMH acquired the data. AMH and ZMS analyzed and interpreted the data. AMH and ZJZ drafted the manuscript. ZJZ and HPL critically revised the manuscript for valuable intellectual content. $\mathrm{AMH}, \mathrm{AMH}$, and ZMS performed statistical analysis. All authors read and approved the final manuscript.

Open Access This article is licensed under a Creative Commons Attribution-NonCommercial 4.0 International License, which permits any non-commercial use, sharing, adaptation, distribution and reproduction in any medium or format, as long as you give appropriate credit to the original author(s) and the source, provide a link to the Creative Commons licence, and indicate if changes were made. The images or other third party material in this article are included in the article's Creative Commons licence, unless indicated otherwise in a credit line to the material. If material is not included in the article's Creative Commons licence and your intended use is not permitted by statutory regulation or exceeds the permitted use, you will need to obtain permission directly from the copyright holder. To view a copy of this licence, visit http://creativecommons.org/licenses/by-nc/4.0/.

\section{References}

1. Chanques G, Jaber S, Barbotte E, Violet S, Sebbane M, Perrigault $\mathrm{PF}$, et al. Impact of systematic evaluation of pain and agitation in an intensive care unit. Crit Care Med. 2006;34(6):1691-9. https:// doi.org/10.1097/01.Ccm.0000218416.62457.56.

2. Skrobik Y, Ahern S, Leblanc M, Marquis F, Awissi DK, Kavanagh BP. Protocolized intensive care unit management of analgesia, sedation, and delirium improves analgesia and subsyndromal delirium rates. Anesth Analg. 2010;111(2):451-63. https://doi. org/10.1213/ANE.0b013e3181d7e1b8.

3. Sacerdote P, Bianchi M, Gaspani L, Manfredi B, Maucione A, Terno G, et al. The effects of tramadol and morphine on immune responses and pain after surgery in cancer patients. Anesth Analg. 2000;90(6):1411-4. https://doi.org/10.1097/00000539-20000 6000-00028.

4. Paulozzi LJ, Budnitz DS, Xi Y. Increasing deaths from opioid analgesics in the United States. Pharmacoepidemiol Drug Saf. 2006;15(9):618-27. https://doi.org/10.1002/pds.1276.

5. Bohnert AS, Valenstein M, Bair MJ, Ganoczy D, McCarthy JF, Ilgen MA, et al. Association between opioid prescribing patterns and opioid overdose-related deaths. JAMA. 2011;305(13):131521. https://doi.org/10.1001/jama.2011.370.

6. Ray WA, Chung CP, Murray KT, Hall K, Stein CM. Prescription of long-acting opioids and mortality in patients with chronic noncancer pain. JAMA. 2016;315(22):2415-23. https://doi.org/ 10.1001/jama.2016.7789.

7. Caspi O, Naami R, Halfin E, Aronson D. Adverse dose-dependent effects of morphine therapy in acute heart failure. Int J Cardiol. 2019;293:131-6. https://doi.org/10.1016/j.ijcard.2019.06.015.

8. von Oelreich E, Eriksson M, Brattström O, Sjölund KF, Discacciati A, Larsson E, et al. Risk factors and outcomes of chronic opioid use following trauma. Br J Surg. 2020;107(4):413-21. https://doi.org/10.1002/bjs.11507.

9. Bellani G, Laffey JG, Pham T, Fan E, Brochard L, Esteban A, et $a l$. Epidemiology, patterns of care, and mortality for patients with acute respiratory distress syndrome in intensive care units in 50 countries. JAMA. 2016;315(8):788-800. https://doi.org/10.1001/ jama.2016.0291.

10. Auriemma CL, Zhuo H, Delucchi K, Deiss T, Liu T, Jauregui $\mathrm{A}$, et al. Acute respiratory distress syndrome-attributable mortality in critically ill patients with sepsis. Intensive Care Med. 2020;46(6):1222-31. https://doi.org/10.1007/ s00134-020-06010-9.

11. Matthay MA, Zemans RL, Zimmerman GA, Arabi YM, Beitler JR, Mercat A, et al. Acute respiratory distress syndrome. Nat Rev Dis Primers. 2019;5(1):18. https://doi.org/10.1038/ s41572-019-0069-0.

12. Barr J, Fraser GL, Puntillo K, Ely EW, Gélinas C, Dasta JF, et al. Clinical practice guidelines for the management of pain, agitation, and delirium in adult patients in the intensive care unit. Crit Care Med. 2013;41(1):263-306. https://doi.org/10. 1097/CCM.0b013e3182783b72.

13. Devlin JW, Skrobik Y, Gélinas C, Needham DM, Slooter AJC, Pandharipande PP, et al. Clinical practice guidelines for the prevention and management of pain, agitation/sedation, delirium, immobility, and sleep disruption in adult patients in the ICU. Crit Care Med. 2018;46(9):e825-73. https://doi.org/10.1097/ ccm.0000000000003299.

14. Johnson AEW, Pollard TJ, Lu S, Lehman LH, Feng M, Ghassemi M, et al. MIMIC-III, a freely accessible critical care database. Sci Data. 2016;3:160035. https://doi.org/10.1038/sdata. 2016.35.

15. Pollard TJ, Johnson AEW, Raffa JD, Celi LA, Mark RG, Badawi O. The eICU Collaborative Research Database, a freely available multi-center database for critical care research. Sci Data. 2018;5:180178. https://doi.org/10.1038/sdata.2018.178.

16. Thompson BT, Chambers RC, Liu KD. Acute respiratory distress syndrome. N Engl J Med. 2017;377(6):562-72. https://doi. org/10.1056/NEJMra1608077. 
17. Gajic O, Dabbagh O, Park PK, Adesanya A, Chang SY, Hou P, et al. Early identification of patients at risk of acute lung injury: evaluation of lung injury prediction score in a multicenter cohort study. Am J Respir Crit Care Med. 2011;183(4):462-70. https://doi.org/10.1164/rccm.201004-0549OC.

18. Sottile PD, Kiser TH, Burnham EL, Ho PM, Allen RR, Vandivier RW, et al. An observational study of the efficacy of Cisatracurium compared with vecuronium in patients with or at risk for acute respiratory distress syndrome. Am J Respir Crit Care Med. 2018;197(7):897-904. https://doi.org/10.1164/rccm. 201706-1132OC.

19. Quan H, Sundararajan V, Halfon P, Fong A, Burnand B, Luthi JC, et al. Coding algorithms for defining comorbidities in ICD-9-CM and ICD-10 administrative data. Med Care. 2005;43(11):1130-9. https://doi.org/10.1097/01.mlr.00001 82534.19832.83.

20. Knaus WA, Wagner DP, Draper EA, Zimmerman JE, Bergner M, Bastos PG, et al. The APACHE III prognostic system. Risk prediction of hospital mortality for critically ill hospitalized adults. Chest. 1991;100(6):1619-36. https://doi.org/10.1378/chest.100.6. 1619.

21. Ho D, Imai K, King G, Stuart EA. Matching as nonparametric preprocessing for reducing model dependence in parametric causal inference. Political Anal. 2007;15:199-236. https://doi. org/10.18637/jss.v042.i08, https://doi.org/10.1093/pan/mpl013.

22. Buuren S, Groothuis-Oudshoorn C. MICE: multivariate imputation by chained equations in R. J Stat Softw. 2011. https://doi.org/ 10.18637/jss.v045.i03.

23. von Elm E, Altman DG, Egger M, Pocock SJ, Gotzsche PC, Vandenbroucke JP. The strengthening the reporting of observational studies in epidemiology (STROBE) statement: guidelines for reporting observational studies. Lancet. 2007;370(9596):1453-7. https://doi.org/10.1016/s0140-6736(07)61602-x.

24. Algera MH, Kamp J, van der Schrier R, van Velzen M, Niesters $\mathrm{M}$, Aarts L, et al. Opioid-induced respiratory depression in humans: a review of pharmacokinetic-pharmacodynamic modelling of reversal. Br J Anaesth. 2019;122(6):e168-79. https://doi. org/10.1016/j.bja.2018.12.023.

25. Lee-Iannotti J, Parish JM. The epidemic of opioid use: implications for the sleep physician. J Clin Sleep Med. 2014;10(6):645-6. https://doi.org/10.5664/jcsm.3790.

26. Yue HJ, Guilleminault C. Opioid medication and sleep-disordered breathing. Med Clin North Am. 2010;94(3):435-46. https://doi. org/10.1016/j.mena.2010.02.007.

27. Al-Hashimi M, Scott SW, Thompson JP, Lambert DG. Opioids and immune modulation: more questions than answers. Br J Anaesth. 2013;111(1):80-8. https://doi.org/10.1093/bja/aet153.

28. Bilfinger TV, Fimiani C, Stefano GB. Morphine's immunoregulatory actions are not shared by fentanyl. Int J Cardiol.
1998;64(Suppl 1):S61-6. https://doi.org/10.1016/s0167-5273(98) 00037-0.

29. Weldon ER, Ariano RE, Grierson RA. Comparison of fentanyl and morphine in the prehospital treatment of ischemic type chest pain. Prehosp Emerg Care. 2016;20(1):45-51. https://doi.org/10. 3109/10903127.2015.1056893.

30. Gurbet A, Goren S, Sahin S, Uckunkaya N, Korfali G. Comparison of analgesic effects of morphine, fentanyl, and remifentanil with intravenous patient-controlled analgesia after cardiac surgery. J Cardiothorac Vasc Anesth. 2004;18(6):755-8. https://doi.org/10. 1053/j.jvca.2004.08.014.

31. Murphy GS, Szokol JW, Marymont JH, Avram MJ, Vender JS. The effects of morphine and fentanyl on the inflammatory response to cardiopulmonary bypass in patients undergoing elective coronary artery bypass graft surgery. Anesth Analg. 2007;104(6):1334-42. https://doi.org/10.1213/01.ane.0000264108.47280.f5.

32. Morgan MM, Tran A, Wescom RL, Bobeck EN. Differences in antinociceptive signalling mechanisms following morphine and fentanyl microinjections into the rat periaqueductal gray. Eur J Pain. 2020;24(3):617-24. https://doi.org/10.1002/ejp.1513.

33. Kanbara T, Nakamura A, Shibasaki M, Mori T, Suzuki T, Sakaguchi G, et al. Morphine and oxycodone, but not fentanyl, exhibit antinociceptive effects mediated by G-protein inwardly rectifying potassium (GIRK) channels in an oxaliplatin-induced neuropathy rat model. Neurosci Lett. 2014;580:119-24. https://doi.org/ 10.1016/j.neulet.2014.08.005.

34. Molina-Martínez LM, González-Espinosa C, Cruz SL. Dissociation of immunosuppressive and nociceptive effects of fentanyl, but not morphine, after repeated administration in mice: fentanylinduced sensitization to LPS. Brain Behav Immun. 2014;42:60-4. https://doi.org/10.1016/j.bbi.2014.06.011.

35. Esteban A, Anzueto A, Frutos F, et al. Characteristics and outcomes in adult patients receiving mechanical ventilation: a 28-day international study. JAMA. 2002;287(3):345-55.

36. Munshi L, Del Sorbo L, Adhikari NKJ, et al. Prone position for acute respiratory distress syndrome. a systematic review and metaanalysis. Ann Am Thorac Soc 2017;14(Supplement_4):S280-8.

37. Conti G, Ranieri VM, Costa R, et al. Effects of dexmedetomidine and propofol on patient-ventilator interaction in difficult-to-wean, mechanically ventilated patients: a prospective, open-label, randomised, multicentre study. Crit Care. 2016;20(1):206. 